\title{
Ultrasound-guided hydrodissection of sural nerve for foot pain- A
} case report

\author{
Jeshnu Tople ${ }^{1}$ and Deepjit Bhuyan ${ }^{1}$ \\ ${ }^{1}$ Datta Meghe Institute of Medical Sciences - Wardha Campus
}

March 17, 2021

\begin{abstract}
Sural nerve entrapment is an impor $\neg$ tant but infrequent cause of pain. The sural nerve provides sensation to the posterolateral aspect of the leg, lateral foot and fifth toe. We present a case of sural nerve entrapment that was effectively treated by percutaneous ultrasound guided hydrodissection.
\end{abstract}

\section{Ultrasound-guided hydrodissection of sural nerve for foot pain- A case report}

\begin{abstract}
:-
Background-Sural nerve entrapment is an important but infrequent cause of pain. The sural nerve provides sensation to the posterolateral aspect of the leg, lateral foot and fifth toe. Sural nerve entrapment can be challenging to treat and can cause significant limitation. We present a case of sural nerve entrapment resistant to conservative management that was effectively treated by percutaneous ultrasound guided hydrodissection of the sural nerve.

Case report - A 57 year old male came with complaints of pain and tingling sensation on both lower limbs with $50 \%$ decrease in sensation to touch (right $>$ left) in lateral aspect of both foot. The patient had tried several conservative modalities with no success. We performed percutaneous ultrasound guided hydrodissection of the sural nerve and the patient reported complete improvement in his pain.

Conclusion: Percutaneous ultrasound guided hydrodissection of the sural nerve, is a safe and effective treatment for patients with sural nerve entrapment that does not respond to conservative therapy. However, studies are needed to elucidate its effectiveness and safety profile.
\end{abstract}

Key words- sural nerve entrapment, ultrasound guided hydrodissection, foot pain, tingling of foot

\section{INTRODUCTION :}

Pain, tingling and numbness on the lateral aspect of the foot are common clinical presentations. Lateral foot pain can be due to various causes including, but not limited to, prolapsed intervertebral disc (PIVD), myofascial pain syndrome like piriformis and entrapment neuropathy like sural nerve, popliteal nerve. ${ }^{(1)}$ Sural nerve entrapment is relatively a rare cause and is often missed. It may present as calf pain, pain over the postero-lateral side of the distal third of the leg, as well as over the lateral aspect of the foot and fifth toe. Sural nerve entrapment can be managed by conservative treatment, interventional treatment and surgery. Interventional treatment can be in the form of hydrodissection, plasma rich platelet (PRP) treatment and radio frequency ablation. We report a case of 57 year old male of sural nerve entrapment successfully treated with hydrodissection.

\section{CASE REPORT:}


A 57 year old male presented to us with the complaints of pain, tingling, electric shock like and burning sensation over lateral aspect of right foot since 2 years. Numerical rating scale (NRS) pain score was 2-3 initially, which progressed over a period of 2 years to score of 7-8. The pain detect tool score was 14 at the time of presentation to us. The patient had been prescribed conservative treatments like vitamin B6 and B12, calcium, gabapentin, nortriptyline and physiotherapy over a period of 2 years by various physicians, though a definite diagnosis was not established. These treatments were unsuccessful in alleviating the pain. The patient was then referred to us. On examination, the patient had about $50 \%$ decrease in sensation to touch over lateral aspect of right foot as compared to the left foot; Tinel's sign was positive behind the right lateral malleolus; motor examination and reflexes were normal. Magnetic resonance imaging (MRI) of the spine was apparently normal. Nerve conduction study showed right lower limb sensory neuropathy. Based on these findings, provisional diagnosis of sural nerve entrapment was established. Patient was prescribed Carbamazepine $200 \mathrm{mg}$ three times a day and Duloxetine with an initial dose of $20 \mathrm{mg}$ twice daily which was gradually increased to $30 \mathrm{mg}$ twice daily. However there was no improvement of symptoms. So discussion was done with the patient about interventional treatment modalities and it was decided to go with ultrasoundguided hydrodissection of sural nerve.

Sural nerve imaging was performed with $12 \mathrm{MHz}$ linear transducer with the patient in the supine position with flexion and adduction of the hip and flexion of knee. Images were obtained in short-axis and long-axis views at the area of entrapment i.e. behind the right lateral malleolus. There was some thickening of fascicles behind the right lateral malleolus compared to the contralateral leg.

Strict aseptic technique was observed during the procedure. The skin over the sural nerve was prepared with povidone-iodine antiseptic solution. The skin and the subcutaneous tissues were infiltrated with $1 \%$ Lidocaine. Then a 24 -gauge needle attached to a syringe containing $10 \mathrm{~mL} 0.9 \%$ normal saline was advanced through the skin and subcutaneous tissues up to the nerve sheath under real-time ultrasound imaging. Under real-time ultrasound guidance, hydrodissection was performed with $0.9 \%$ normal saline and after that perineural injection of $1 \%$ Lidocaine and Methylprednisolone $40 \mathrm{mg} 4 \mathrm{~mL}$ was done. The needle was then removed. The area was massaged both proximally and distally to enhance hydrodissection release. The patient tolerated the procedure well with virtually no discomfort. The patient was followed up on day 7,1 month, 2 months and 3 months after the intervention. The patient reported relief of symptoms on day 1 except for mild pain of needle prick. On subsequent follow up patient had complete relief of pain with NRS pain score of 0 to 1 and pain detect tool score was zero. No side effect or complication was encountered over follow up to 3 months. 


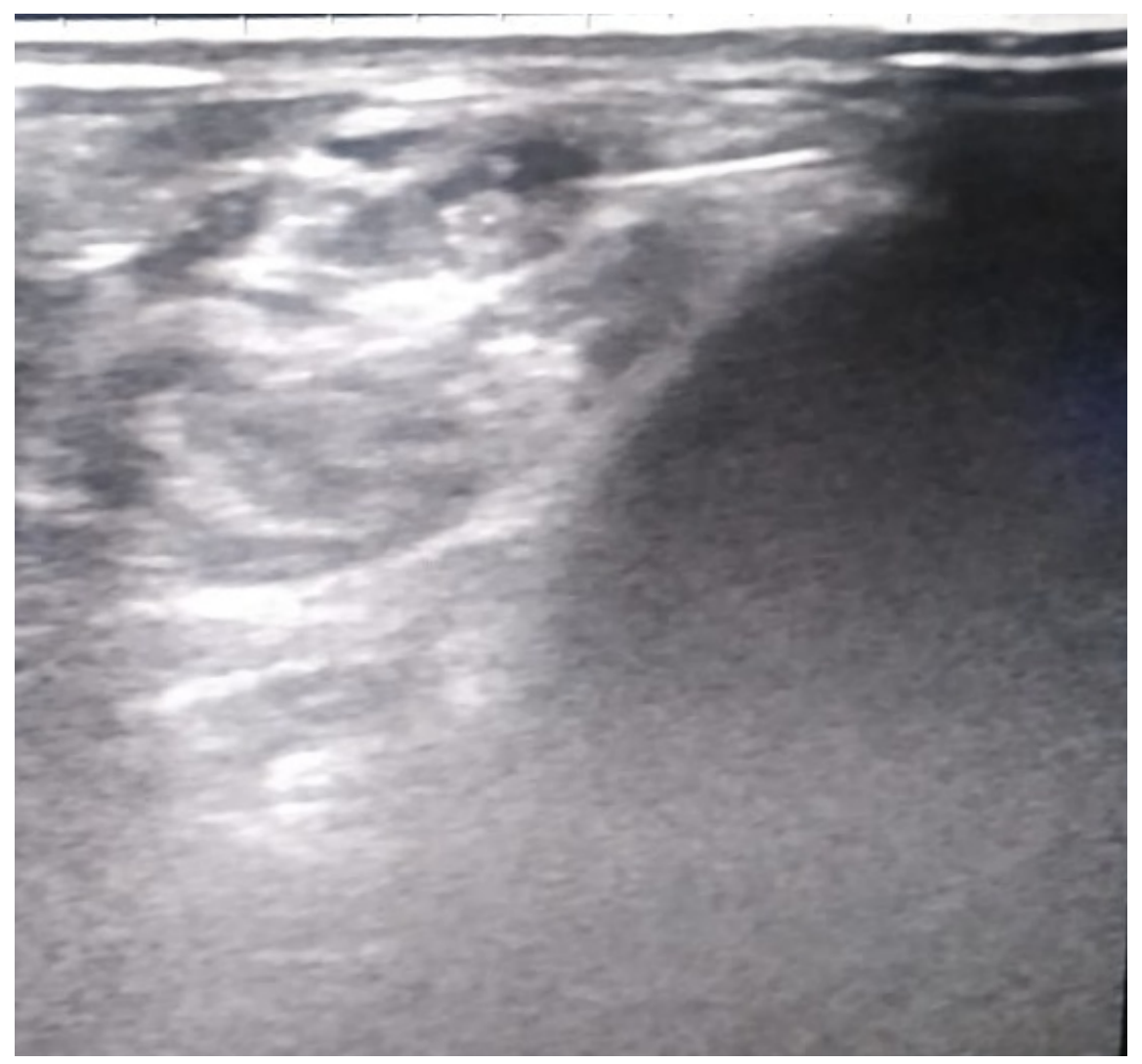




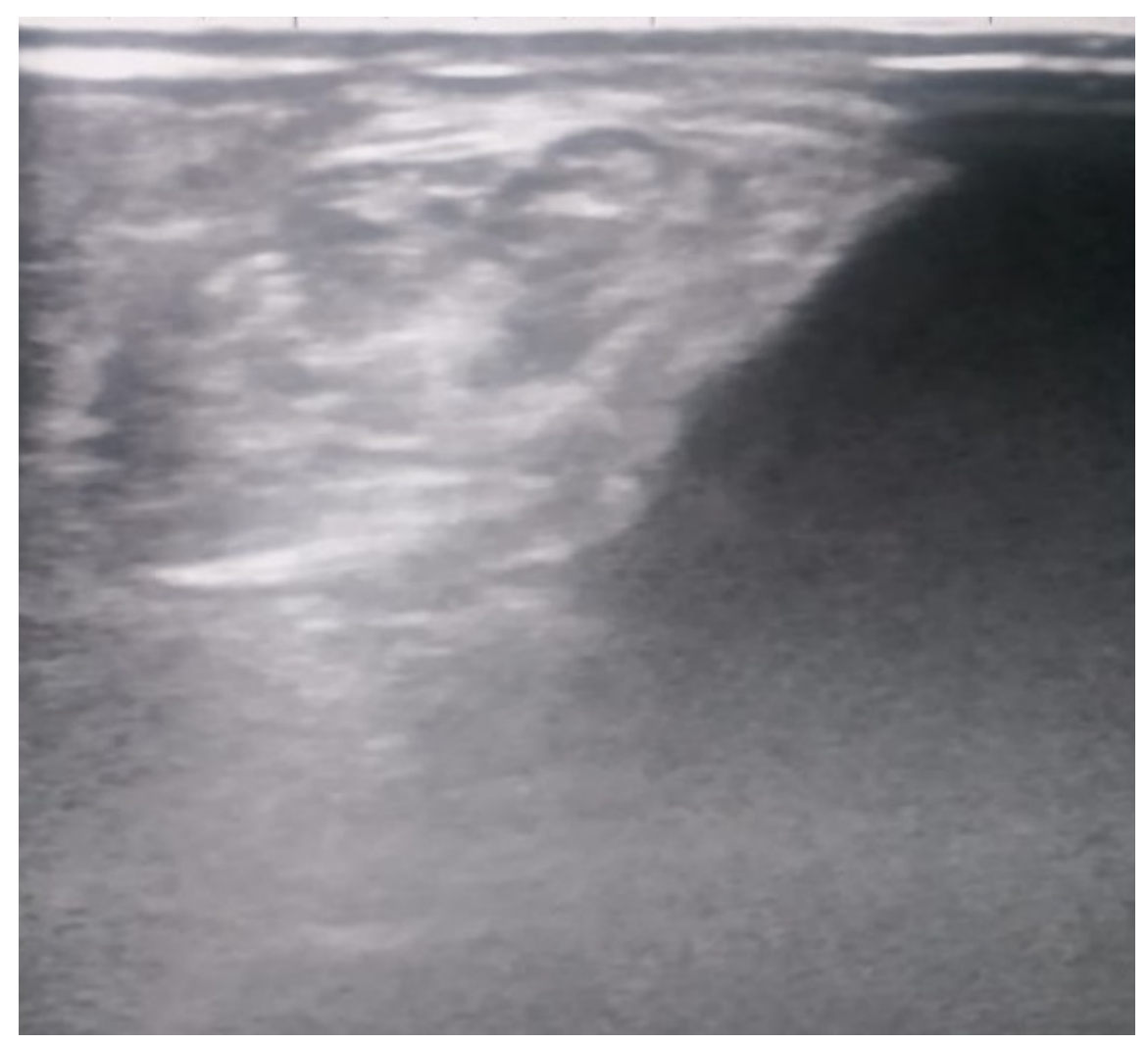

Figure 1- Ultrasound guided view of the sural nerveFigure 2 - Ultrasound guided view of hydrodissection of sural nerve

\section{DISCUSSION :}

The sural nerve is a sensory nerve of lower limb that supplies posterolateral surface of leg and lateral part of dorsum of foot and heel. It is formed by communication of medial sural cutaneous nerve (MSCN) and peroneal communicating nerve (PCN). MSCN arise from tibial nerve in popliteal fossa. PCN is a branch directly from common peroneal nerve or from lateral sural cutaneous nerve. Sural nerve can be just a continuation of MSCN when there is no communication between MSCN and PCN. ${ }^{(2,3,4)}$ 


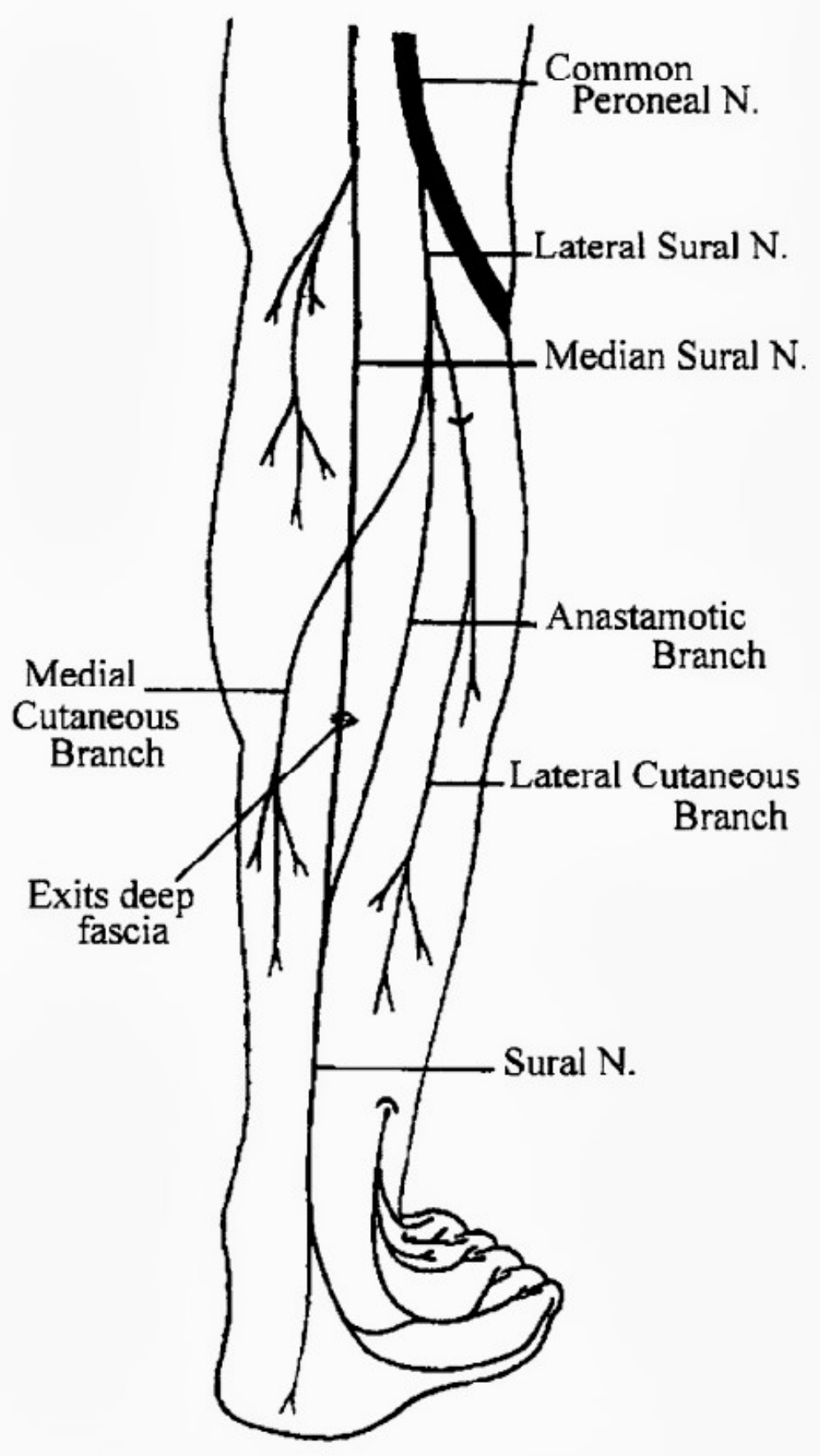

Figure 3 - Course of the sural nerve. ${ }^{(5)}$

Sural nerve may get affected as a result of compression, direct trauma, inversion ankle sprain or adverse neurodynamics. Compression of the nerve can be due to thickening of crural fascia; various mass lesions such as a Bakers Cyst, ganglion, lipomas or myositis ossificans at the level of gastrocnemius aponeurosis; scar tissue beneath the deep fascia of the gastrocnemius, peroneal nerve sheath degeneration or Achilles 
paratendinitis; and extrinsic causes such as ski boots, heel straps, casts and tightly laced boots. Direct trauma responsible for sural nerve neuropathy may include fibular fracture, fracture of the fifth metatarsal, gastrocnemius muscle injury, achilles tendon rupture or surgical induced damage.

The sural nerve can become entrapped anywhere along its course in the lower extremity. Entrapment involving the sural nerve typically occurs at the musculotendinous junction of the gastrocnemius muscle and the Achilles tendon within the calf, as the nerve travels through a fibrous arcade (which has been termed the superficial sural aponeurosis), at the ankle or in the lateral foot near the base of the fifth metatarsal. ${ }^{(6)}$ The tension is worsened during passive forcible plantar flexion and inversion of the foot increases tension in the sural nerve as the nerve is tightly adherent to the surrounding fascia. ${ }^{(7)}$ Entrapment of the sural nerve can also be due to post-traumatic scar tissue beneath the deep fascia of gastrocnemius, peroneal nerve sheath degeneration, calcaneocubiod joint capsule degeneration and Achilles tendonitis. ${ }^{(8)}$

\section{Hosted file}

image4.emf available at https://authorea.com/users/402158/articles/514037-ultrasound-guidedhydrodissection-of-sural-nerve-for-foot-pain-a-case-report

Figure 4 - Sites of entrapment of the sural nerve.A- junction of the gastrocnemius muscle and Achilles tendon,B- lateral ankle, C- lateral foot ${ }^{(6)}$

The treatment of sural nerve neuropathy can be conservative, interventional or surgical. Surgical treatment is required when the removal of space-occupying masses (ganglion/lipoma) is necessary. Conservative treatments include physiotherapy, non-steroidal anti-inflammatory drugs, vitamin B6 \& B12, tricyclic antidepressants, antiepileptics, or topical anesthetics. Interventional treatments include radiofrequency ablation of the nerve, plasma rich platelet (PRP) infiltration and percutaneous ultrasound guided hydrodissection. ${ }^{(5,9-14)}$

Sural nerve hydrodissection is one of the least studied interventional techniques but it is considered to be a useful technique to treat neuropathic pain caused by nerve entrapment. In nerve hydrodissection ultrasound-guided fluid is injected to separate nerve from the surrounding structure, usually the fascia, which is believed to constrict or irritate the nerve either during movement or at rest. ${ }^{(15,16)}$ The advantage of nerve hydrodissection is separation of nerves from the surrounding soft tissue with fluid (hydro) and with the fluid flushing and the release of pressure on the nervi nervorum outside the epineurium. ${ }^{(16)}$ These nervi nervorum are the free nerves supplying the main nerve and regulate the function and discharge of the main nerve. ${ }^{(14)}$ Real time ultrasonography is usually the choice of imaging technique in nerve hydrodissection to guide needles and fluid injection. Traditionally, the fluid used for hydrodissection is a large volume of $0.9 \%$ normal saline or $5 \%$ dextrose. Along with that a small volume of steroid and local anesthetic solution is also injected to reduce the pain. ${ }^{(16)}$ We performed hydrodissection using $0.9 \%$ normal saline $10 \mathrm{~mL}$, methylprednisolone $40 \mathrm{mg}$ and $1 \%$ lidocaine at the entrapment site, which was behind the lateral malleolus due to fasicular hypertrophy of the peroneal retinaculam. The outcome was excellent during follow-up up to 3 months.

\section{CONCLUSION:}

Sural nerve entrapment behind the lateral malleolus should be considered as a potential etiology in patient presenting with sensory disturbances along the lateral aspect of foot or ankle. Ultrasound imaging adds in formation of the diagnosis in entrapment. Moreover real time ultrasound is useful for treatment by hydrodissection technique. Hydrodissection is an interventional procedure that is efficacious and cost effective with good outcome and hence is an attractive option treatment option for sural entrapment neuropathy. However, sporadic studies for hydrodissection of entrapped sural nerve mandate additional studies to elucidate effectiveness and safety profile of this technique.

\section{REFERENCES:}

1. Boyajian-O'Neill, L.A, Mcclain, R.L, Coleman, M.K, Thomas, P.P. Diagnosis and Management of Piriformis Syndrome: An Osteopathic Approach. J Am Osteopath Assoc. 2008;108(11): 657-664. 
2. Bianchi, S, Droz, L, Deplain, C.H. Ultrasonograph y of the Sural Nerve - Normal and Pathologic Appearances. J Ultrasound Med. 2018;37(05): 1257-1265.

3. Paraskevas, G.K, Natsis, K. Fascial entrapment of the sural nerve and its clinical relevance. Anatomy and Cell Biology. 2014;47(10): 144-147.

4. Kavyashree, A.N, Subhash, L.P. Anatomical Variations in Formation of Sural Nerve in Adult Indian Cadavers. Journal of Clinical and Diagnostic Research. 2013;07(09): 1838-1841.

5. Blackshear, M.B, Lutz, G.E, O'brien, S.J. Archives of Physical Medicine and Rehabilitation. Sural Nerve Entrapment After Injury to the Gastrocnemius: A Case Report. 1999;80(05): 604-605.

6. Brown M.N., Pearce B.S., Vanetti T.K. (2016) Sural Nerve Entrapment. In: Trescot A.M. (eds) Peripheral Nerve Entrapments. Springer, Cham.

7. Miniato MA, Nedeff N. Anatomy, Bony Pelvis and Lower Limb, Sural Nerve. [Updated 2020 Aug 15]. In: StatPearls [Internet]. Treasure Island (FL): StatPearls Publishing; 2021 Jan-

8. Seema, S.R. Study of Sural Nerve Complex in Human Cadavers. ISRN Anatomy. 2013;2013(1): 1-7.

9. Fader, R.R, Mitchell, J.J. Percutaneous Ultrasound-Guided Hydrodissection of a Symptomatic Sural Neuroma. Orthopedic. 2015;38(11): 1046-1050.

10. Trescot, A, Brown, M. Peripheral nerve entrapment, hydrodissection, and neural regenerative strategies. Techniques in Regional Anesthesia [amp] Pain Management. 2016;19(19): 85-93.

11. Eker, H.E, Cok, O.Y. Management of Neuropathic Pain with Methylprednisolone at the Site of Nerve Injury. Pain Medicine. 2012;13(13): 443-451.

12. Malahias, M.A, Chytas, D. Platelet-rich plasma guided injections: clinical application in peripheral neuropathies. Frontiers in surgery. 2014;01(41): 1-4.

13. Abd-Elsayed, A, Jackson, M, Plovanich, E. Pulsed Radiofrequency Ablation for Treating Sural Neuralgia. Ochsner Journal. 2018;18(01): 88-90.

14. Jia, X, Peters, P.G, Schon, L. The use of platelet rich plasma in the management of foot and ankle conditions. Operative Techniques in Sports Medicine. 2011;19(03): 177-184.

15. Watanabe, K, Tokumine, J, Lefor, A.K, Moriyama, K, Yorozu, T. Ultrasound-Guided Hydrodissection of an Entrapped Saphenous Nerve After Lower Extremity Varicose Vein Stripping: A Case Report. A A Pract. 2020;14(01): 28-30.

16. Lam, S.K.H, Hung, C.Y, Chiang, Y.P, Moriyama, K, Yorozu, T. Ultrasound-Guided Nerve Hydrodissection for Pain Management: An Updated Review of Anatomy and Techniques. Journal of pain and research. 2020;13(01): 1957-1968. 